\title{
A Lux-like Quorum Sensing System in the Extreme Acidophile Acidithiobacillus ferrooxidans
}

\author{
MARIELLA RIVAS ${ }^{1}$, MICHAEL SEEGER $^{2}$, DAVID S HOLMES $^{3}$ and \\ EUGENIA JEDLICKI ${ }^{*}$
}

\begin{abstract}
${ }^{1}$ Institute of Cellular and Molecular Biology (ICBM), University of Chile, Santiago, Chile, Laboratory of Molecular Microbiology and Environmental Biotechnolgy, Dept. of Chemistry, Federico Santa María Technical University and ${ }^{3}$ Laboratory of Bioinformatics and Genome Biology, Millennium Institute of Fundamental and Applied Biology and Andrés Bello University, Santiago, Chile.
\end{abstract}

\begin{abstract}
The genome of the acidophilic, proteobacterium Acidithiobacillus ferrooxidans, contains linked but divergently oriented genes, termed $a f e I$ and $a f e R$, whose predicted protein products are significantly similar to the LuxI and LuxR families of proteins. A possible promoter and Lux box are predicted upstream of afeI. A cloned copy of afeI, expressed in E. coli, encodes an enzyme that catalyzes the production of a diffusible compound identified by gas chromatography and mass spectrometry as an unsubstituted $N$-acyl homoserine lactone (AHL) of chain length $\mathrm{C}_{14}$. This $\mathrm{AHL}$ can be detected by a reporter strain of Sinorhizobium meliloti $\mathrm{Rm} 41$ suggesting that it is biologically active. The reporter strain also responds to extracts of the supernatant of A.ferrooxidans grown to early stationary phase in sulfur medium indicating that a diffusible AHL is produced by this microorganism. Semi-quantitative RT-PCR experiments indicate that $a f e I$ and $a f e R$ are expressed maximally in early stationary phase and are more expressed when A.ferrooxidans is grown in sulfur- rather than iron-containing medium. Given the predicted amino acid sequence and functional properties of AfeI and AfeR it is proposed that A.ferrooxidans has a quorum sensing system similar to the LuxI-LuxR paradigm.
\end{abstract}

Key terms: A.ferrooxidans, quorum-sensing, LuxI and LuxR, Lux box, homoserine lactone.

\section{INTRODUCTION}

Quorum sensing is a mechanism for regulating gene expression in response to changes in cell density in bacterial populations. One type of quorum sensing mechanism, widely used by proteobacteria, involves the production of an $N$-acyl homoserine lactone (AHL) autoinducer. AHL is synthesized from $\mathrm{S}$-adenosylmethionine (SAM) (Hanzelka and Greenberg, 1997) via the activity of LuxI-type acyl synthase. AHL molecules diffuse through the bacterial membrane and when a critical extra-cellular threshold concentration is reached, they are detected by intracellular LuxR-type proteins that can regulate not only the expression of luxI but also other target genes involved in a variety of behavioral responses (reviewed in
Pappas et al., 2004). In many instances it has been shown that LuxR regulates gene expression by binding to upstream consensus sequences, termed Lux-boxes, upregulating gene transcription. The types of responses regulated by the quorum sensing genetic circuits include bioluminescence (Nealson, 1999), the horizontal transfer of DNA (Winans et al., 1999; Whitehead et al., 2001), the formation of biofilms (Conway et al., 2002) and the production of pathogenetic factors, antibiotics and other secondary metabolites (Pesci and Iglewski, 1999; reviewed in Miller and Bassler, 2001)

Acidithiobacillus ferrooxidans, formerly called Thiobacillus ferrooxidans (Kelly and Wood, 2000) is a chemolithoautotrophic, $\gamma$ proteobacterium that obtains energy and electrons by the oxidation of reduced sulfur

\footnotetext{
*Corresponding author. Eugenia Jedlicki, Institute of Cellular and Molecular Biology (ICBM), Faculty of Medicine, Av. Independencia 1027, Santiago, Chile, Fax: 56-2-735-5580, Telephone: 56-2-978-6258, Email: ejedlick@med.uchile.cl
} 
compounds to sulfate or FeII to FeIII. It is a mesophilic, facultative aerobe that fixes atmospheric $\mathrm{CO}_{2}$ and $\mathrm{N}_{2}$ to provide cellular $\mathrm{C}$ and $\mathrm{N}$. It thrives in extremely acidic conditions ( $\mathrm{pH} \mathrm{1-2)}$ and is often confronted with high concentrations of metals. These multiple challenges make it an excellent choice for understanding microbial physiology in extreme environments.

A. ferrooxidans is a member of a consortium of microorganisms found in bioleaching operations and other naturally low $\mathrm{pH}$ environments (reviewed in Rawlings, 2002) and is known to occur in biofilms (Gehrke et al., 1998, Schippers and Sand, 1999). A knowledge of its role in the development and maintenance of biofilms is important for generating a comprehensive description of its role in mineral leaching and environmentally associated processes. Quorum sensing processes have been invoked in the establishment and maintainance of biofilms in a number of environmental bacteria (Bollinger et al., 2001; Conway et al., 2002; Huber, et al., 2001), but only two preliminary reports of the presence of potential quorum sensing genes in $A$. ferrooxidans have been published (Barreto et al., 2003; Farrah et al., 2004). This lack of information provoked the present study.

\section{METHODS}

\section{Bacterial strains, plasmids and media}

Acidithiobacillus ferrooxidans ATCC 23270 was grown in $9 \mathrm{~K}$ salts medium $(\mathrm{pH}$ 2.4) supplemented with elemental sulfur or iron $\left(\mathrm{FeSO}_{4}\right)$ as described by Yates and Holmes (1988). Escherichia coli JM109 was grown in Luria-Bertani (LB) medium. Agrobacterium tumefaciens NT1 was grown in LB medium containing kanamycin 50 $\mu \mathrm{gml}^{-1}$ at $30^{\circ} \mathrm{C}$. Sinorhizobium meliloti Rm41was grown in LB at $30^{\circ} \mathrm{C}$ and $S$. meliloti Rm41 SinI- was grown in LB or MMgly medium (11 g of $\mathrm{Na}_{2} \mathrm{HPO}_{4}, 3 \mathrm{~g}$ of $\mathrm{KH}_{2} \mathrm{PO}_{4}, 0.5 \mathrm{~g} \mathrm{NaCl}, 1 \mathrm{~g}$ of $\mathrm{NH}_{4} \mathrm{Cl}, 5 \mathrm{ml}$ of glycerol, $1 \mathrm{mg}$ of biotin, $27.8 \mathrm{mg}$ of $\mathrm{CaCl}_{2}$, and $246 \mathrm{mg}$ of $\mathrm{MgSO}_{4}$ per liter) at $30^{\circ} \mathrm{C}$ suplemmented with $1 \%(\mathrm{w} / \mathrm{v})$ L-arabinose. Antibiotics were added where appropriate at the following final concentrations

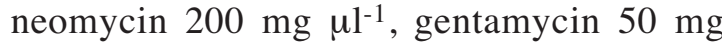
$\mu l^{-1}$. Details of the phenotypes and sources of bacteria are shown in Table I.

TABLE I

Bacterial strains and plasmids used in this study

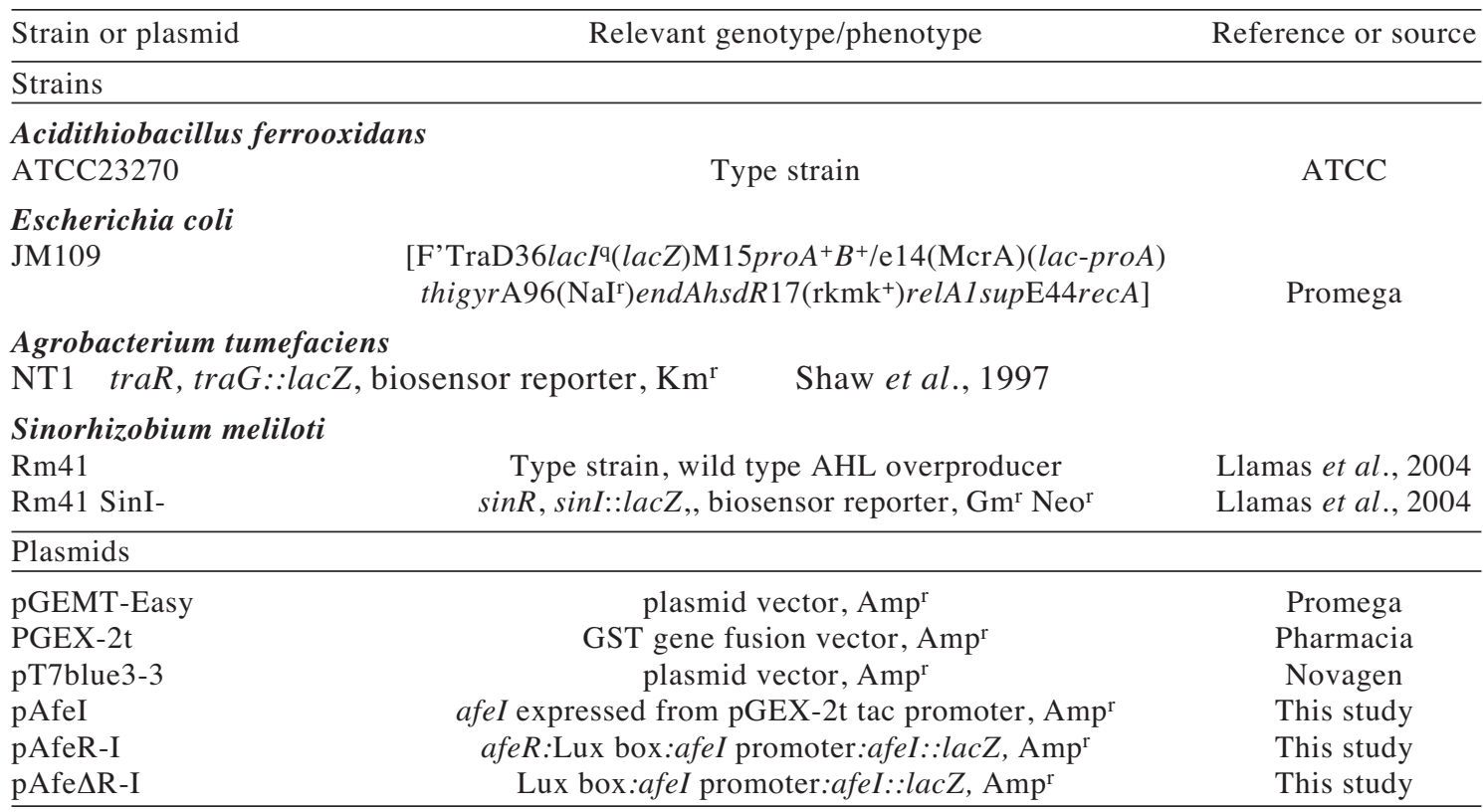




\section{Bioinformatic Analyses}

Candidate protein coding genes were identified in the partial genome sequence of A. ferrooxidans ATCC 23270, made available by The Institute for Genome Research (TIGR, www.tigr.org) using Glimmer (www.tigr.org), Critica (www.ttaxus.com) and BlastX (www.ncbi.nlm.nih.gov), followed by manual curation of the predicted genes to correct errors in start site prediction and identify missing candidate genes. The annotated genome was displayed in the interactive format of Artemis (www.sanger.ac.uk/Software/Artemis). The following bioinformatic programs were used to further characterize candidate genes and their predicted protein products: BlastP and PsiBlast (www.ncbi.nlm.nih.gov), the suite of protein characterization programs available in InterproScan (www.ebi.ac.uk/ interpro), Blocks (www.blocks.fhcrc.org) and ClustalW (www.ebi.ac.uk/ClustalW). Additional protein motif finding programs were used to detect potential helix-turnhelix (npsa-pbil.ibcp.fr/cgi-bin/ primanal_hth.pl) and signal peptide motifs (www.psort.nibb.ac.jp). A candidate LuxR transcription factor binding sites (Lux box) was identified by using a motif model of the LuxR binding site in DNA (McGuire et al., 2000). The putative target was subjected to a cross-species comparison (phylogenetic footprinting) to increase confidence in the prediction using a Gibbs sampling-based motif-detection procedure (McCue et al., 2001; Frazer et al., 2003). Candidate sigma-70 promoters were detected using a neural network program for prokaryotic sigma-70 promoters ( w w w.f r u itfly .org / s e q _ tools / promoter.html).

\section{Purification and identification of $A H L$}

A. ferrooxidans and recombinant E. coli pAfeI early stationary-phase cultures (including cells and supernatants) were extracted with dichloromethane (DCM) at a ratio of 70:30 (culture:DCM) as described (McClean et al., 1997). DCM was removed by rotary evaporation and the residue reconstituted in $100 \mu \mathrm{l}$ DCM for fractionation by sep-pack $C_{18}$ preparative columns. Fractions were eluted with $1 \mathrm{ml}$ in a gradient of methanol in water (20-40-60$75-95 \%, v / v)$. Five fractions (F1-F5) were collected, concentrated to $10 \mathrm{ml}$ and assayed for activity using the AHL assays described above. Samples $(1 \mu \mathrm{l})$ were also injected in the splitless mode into a gas chromatography-mass spectrometry (GC/ MS) system consisting of a Autosystem XL gas chromatograph (Perkin-Elmer, Boston, MA, USA) with a MDN-5 column (Supelco, Bellefonte, PA, USA), coupled to a PerkinElmer Turbo Mass mass spectrometer. Helium served as carrier gas. The mass spectrometer was operated in the electron impact ionization mode at $70 \mathrm{eV}$ as described (Seeger et al., 2001, 2003). The following AHL standards were purchased from Fluka: $N$-hexanoyl-DL-homoserine lactone $\left(\mathrm{AHL}-\mathrm{C}_{6}\right), \quad N$-octanoyl-DLhomoserine lactone (AHL- $\mathrm{C}_{8}$ ) and $N$ tetradecanoyl-DL-homoserine lactone (AHL$\mathrm{C}_{14}$ ).

Isolation of DNA, recombinant DNA techniques, DNA sequencing

DNA was isolated from A. ferrooxidans as described (Barreto et al., 2005). The following standard recombinant DNA techniques: digestion of DNA with restriction enzymes, agarose gel electrophoresis, purification of DNA fragments, PCR amplification of DNA, DNA ligation, plasmid preparation and transformation of $E$. coli, were performed as described (Sambrook et al., 1989). DNA sequencing was carried out by the Sanger dideoxynucleotide method (Sambrook et $a l ., 1989)$. The nucleotide sequence of the afeR-I locus has been assigned the GenBank accession number AY758559.

Construction of recombinant plasmids and analysis of gene expression

AfeI was amplified with Elongase mix (Invitrogen) using genomic DNA as a template with the primers AfeI-4 and AfeI5 (Table II). The resulting amplified DNA was cloned into pGEMT-easy as described 
by the supplier (Promega). AfeI was subcloned pGEMT-easy into pGEX-2t (Amershan Pharmacia Biotech) as described by the suppliers. The resulting plasmid was termed pAfeI. pGEX-2t and pAfeI were separately transformed into $E$. coli JM109 by electroporation and the transformants were grown on LB plates containing ampicillin $100 \mu \mathrm{g} \mathrm{ml}^{-1}$.

Genomic DNA corresponding to the entire predicted afeR, the first $120 \mathrm{bps}$ of afeI and the whole of the intergenic region between these two genes (Fig. 1) was amplified by PCR using the primers AfeR-3 and AfeI-3 (Table II). The resulting amplified DNA was cloned in pT7-Blue3 (Novagen) and subsequently subcloned into pQF50 (Farinha and Kropinski, 1990) in an orientation that places the expression of $l a c Z$ in pQF50 under the control of the putative promoter of afeI. The resulting plasmid was termed pAfeR-I and was introduced into $E$. coli JM109 by electroporation and the transformants were grown in LB plates containing ampicillin $100 \mathrm{mg} \mu \mathrm{l}^{-1}$. In addition, a plasmid similar to pAfeR-I but lacking $A f e R$ was constructed and termed pAfe $\Delta$ R-I.

Promoter activity of pAfeR-I.

Overnight E. coli JM109 (pAfeR-I) cultures were normalized to an optical density at $600 \mathrm{~nm}$ of 0.1 in a volume of $5 \mathrm{ml}$ of LB containing the desired AHL at the desired concentration. Cultures were grown with agitation at $37^{\circ} \mathrm{C}$ for $6 \mathrm{~h}$, and $\beta$ galatosidase activities were determined by the Miller assay as previously described (Quatrini et al., 2005).

\section{AHL reporter plate assays}

E. coli JM109 (pAfeI) was cross-streaked onto X-gal $80 \mathrm{mg} \mu \mathrm{l}^{-1}$, IPTG $40 \mu \mathrm{g} \mathrm{ml^{-1 } \mathrm { LB }}$ medium reporter plates in the presence $A$. tumefaciens NT1 (Shaw et al., 1997) and $S$. meliloti Rm41 SinI- (Llamas et al., 2004) as described by Latifi et al. (1995) and Swift et al. (1997). Plates were incubated overnight at $30^{\circ} \mathrm{C}$. Activation of the lacZ gene in these reporter strains was detected visibly by the production of blue dye. As a negative control, E. coli JM109 lacking afeI but containing the plasmid vector $\mathrm{pGEX}-2 \mathrm{t}$ was also cross-streaked on the same plates. As a positive control, the AHL-producing strain S. meliloti Rm41 was also crossstreaked. The presence of AHL was also detected on solid media by growing $E$. coli (pAfeR-I) on X-Gal medium supplemented with ampicillin $\left(100 \mu \mathrm{g} \mathrm{ml^{-1 }}\right)$ in close proximity to the tester strain. The presence of AHL was observed when E. coli (pAfeRI) turned blue.

TABLE II

PCR and RT-PCR primers used in this study

\begin{tabular}{|c|c|c|}
\hline Gene & Primer name & Sequence $\left(5^{\prime} \text { to } 3^{\prime}\right)^{\mathrm{a}}$ \\
\hline \multirow[t]{5}{*}{ afeI } & afeI-1 & 5’CAGGTTATAACCGGGCCAGCTG \\
\hline & afeI-2 & 5’ATCAGCCGTTTTGCCCCGTG \\
\hline & afeI-3 & 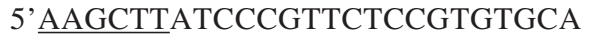 \\
\hline & afeI-4 & 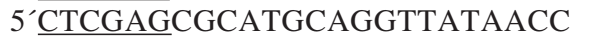 \\
\hline & afeI-5 & 5’GAGCTCGCGGTCCAGATCTATCCA \\
\hline \multirow[t]{3}{*}{ afeR } & afeR-1 & 5'GAGCATCGCCGCCTGCAATA \\
\hline & afeR-1 & 5’AGGCCATCGATCCCACGGTA \\
\hline & afeR-1 & 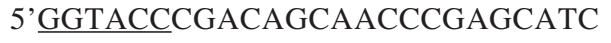 \\
\hline \multirow[t]{2}{*}{ afe 1016} & afe 1016-1 & 5’ATGACAGTGCAGAACGGAAT \\
\hline & afe 1016-1 & 5’AACATATCTATCCATGATAT \\
\hline \multirow[t]{2}{*}{ recA } & recA-1 & 5’CCGCCAACATTTCCCGGACC \\
\hline & recA-1 & 5'ACGCCGAGGTCCACCAGTTC \\
\hline
\end{tabular}

\footnotetext{
a Underlined are added restriction enzyme sites.
} 

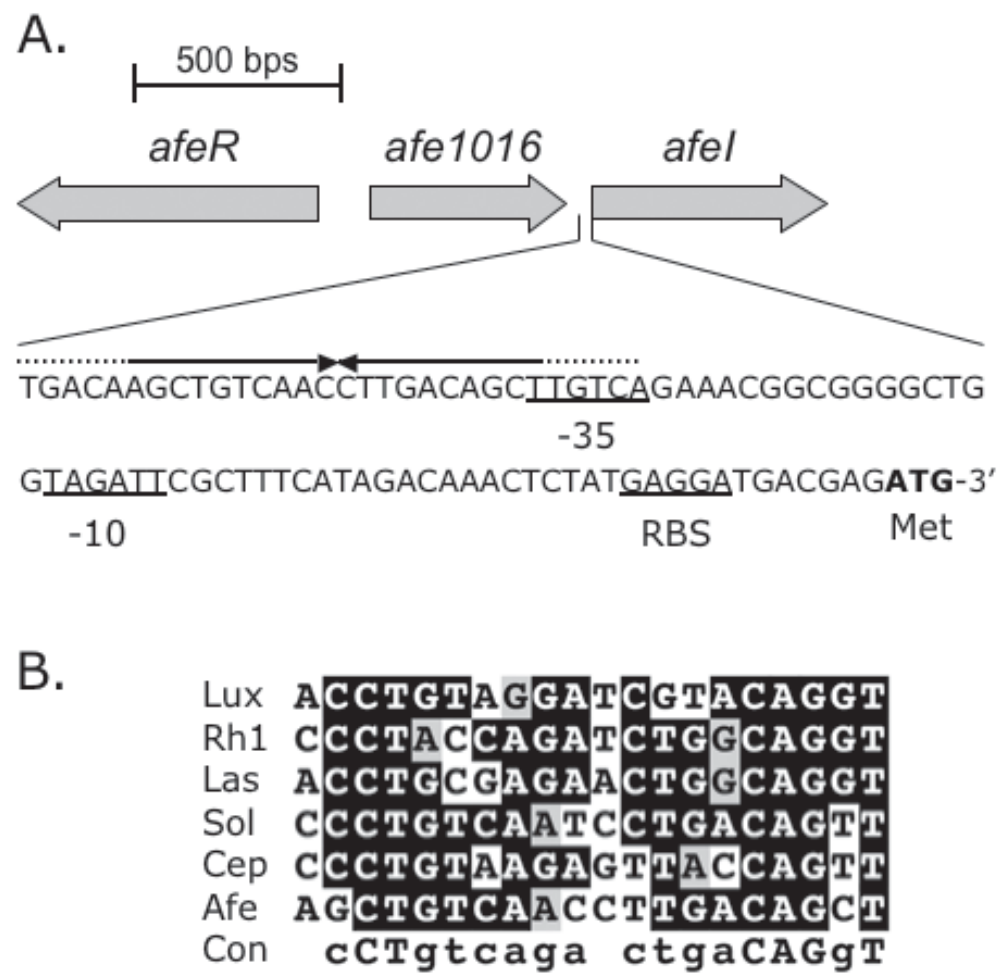

Figure 1. (A) Organization of the predicted genes of the Lux-like quorum sensing locus of $A$. ferrooxidans. Arrows indicate the proposed direction of transcription. The inwardly directed arrows indicate the predicated Lux box and the dotted lines demonstrate the potential extension of the conserved Lux box another 5 bps upstream and downstream. Underlined are predicted -35 and -10 regions of a sigma 70-like promoter and a ribosome binding site (RBS). The predicted initial ATG codon of afeI is indicated. (B) Comparison of the predicted Lux box of A.ferrooxidans (Afe) with other know Lux boxes, shaded nucleotides are the most conserved. Lux = Lux box of Vibrio fisheri (Devine et al., 1988), Rhl = Rhl box, Las = Las box of Pseudomonas aeruginosa (Latifi et al., 1995), Sol = Sol box of Ralstonia solanacearum (Flavier et al., 1997), Cep = Cep box of Burkholderia cepacia (Lewenza et al.,1999). Con = consensus sequence of the Lux boxes derived from the alignment of the six Lux boxes.

In vitro assays of $\beta$-galactosidase production.

A. tumefaciens NT1 and S. meliloti $\mathrm{Rm} 41$ SinI- were grown to $0.5 \mathrm{OD}_{600}$ in 2 $\mathrm{ml}$ of $\mathrm{LB} / \mathrm{MC}$ broth with $1 \%(\mathrm{w} / \mathrm{v}) \mathrm{L}-$ arabinose at $30^{\circ} \mathrm{C}$. The cultures were diluted $1: 10 \mathrm{ml}$ in $\mathrm{Z}$ buffer $(0.06 \mathrm{M}$ $\mathrm{Na}_{2} \mathrm{HPO}_{4}, 0.04 \mathrm{M} \mathrm{KCl}, 0.001 \mathrm{M} \mathrm{MgSO}$, $0.05 \mathrm{M} \beta$-mercaptoethanol) and assayed by the Miller procedure (Miller, 1972) as previously described (Quatrini et al., 2005) to determine Miller units of activity, using o-nitrophenyl- $\beta$-D-galactopyranoside as the substrate. Each sample was assayed in triplicate, and each experiment was repeated at least three times. AHL crude extracts from Rm41 or A. ferrooxidans or recombinant $E$. coli pAfeI or the different synthetic and commercial short and longchain AHLs (see above) were added to the medium at the time of inoculation.

Semi-quantitative RT-PCR measurements of gene expression

Five micrograms of total RNA was isolated from A. ferrooxidans and was reverse transcribed by PCR (RT-PCR) as described previously (Guacucano et al., 2000) using the following DNA primers: afeI-1 and afeI-2 to amplify DNA corresponding to the 
predicted afeI, afeR-1 and afeR-2 corresponding to afeR and recA-1, recA-2 to amplify DNA corresponding to recA (control gene to evaluate constitutive expression) (Table II). Semi-quantitative PCR was carried out by one cycle of incubation at $94^{\circ} \mathrm{C}$ for $1 \mathrm{~min}$, followed by $15,20,25$ and 30 cycles of $90^{\circ} \mathrm{C}$ for $30 \mathrm{~s}$ each, $64^{\circ} \mathrm{C}$ for $1 \mathrm{~min}$, and $72^{\circ} \mathrm{C}$ for $1 \mathrm{~min}$. PCR products were visualized by agarose gel electrophoresis as previously described (Guacucano et al., 2000). Densitometry measurements of DNA were quantitated using Scion Image for Windows software. Appropriate negative and positive controls were included in each RT-PCR experiment as described previously (Guacucano et al., 2000). PCR was performed with up to 100fold dilutions of template to ensure that assays were carried out in the linear range of template concentration. Reproducibility was assessed by performing at least two independent RT reactions for each time point and at least three PCRs using each of these templates.

\section{RESULTS}

Identification and organization of the afeIafe $R$ locus in the genome of A.ferrooxidans

Putative genes, termed afeI and afeR (formerly traI and traR, Barreto et al., 2003), have been detected in the genome of A. ferrooxidans using bioinformatic procedures. The predicted AfeI and AfeR protein products exhibit significant amino acid sequence similarity and conservation of motifs, domains and patterns with the LuxI and LuxR family of proteins, respectively, involved in quorum sensing in many Gram-negative bacteria (Table III) (Pappas et al., 2004).

Predicted characteristics of the genes and protein products of the Lux-like quorum sensing locus of A.ferrooxidans (Gen Bank acc. o AY 758559)

\begin{tabular}{lcccccc}
\hline Gene name & Function assigned & Best BlastP hit & $\%$ similarity & E value & Score & Motif, patter \\
\hline afeI & Autoinducer lactone & Burkholderia & & & & \\
& synthase & pseudomallei & 69 & $1 \mathrm{e}-47$ & 190 & PD002752 \\
& & & & PR01549 \\
& & & & PF00765 \\
& & & SSF55729 \\
& & & IPR001690
\end{tabular}

afe 1016

unknown

Burkholderia

pseudomallei

$51 \quad 1 \mathrm{e}-14$

81

afeR

Burkholderia mallei 62 $7 e-55$

PD000307

PR00038

PF001968

SM00421

PS50043

IPR000729

IPR005143

\footnotetext{
$\%$ similarity $=$ similarity of amino acid sequence. $\mathrm{E}$ value and scores were derived from BlastP. PD $=$ Prodom, $\mathrm{PR}=$ Prosite, $\mathrm{PF}=$ Pfam, $\mathrm{SSF}=$ SuperFamily, $\mathrm{SM}=$ Smart, PS = Profile Scan and IPR = InterproScan.
} 
AfeI and afeR are organized in a divergent fashion, as has been observed in several other bacteria (Stevens and Greenberg, 1997; Lewenza et al., 1999; reviewed in Miller and Bassler, 2001) (Fig. 1A). A conserved hypothetical gene (afe1016, Fig. 1A) of unknown function (Table III) is located in the intergenic region between afeI-afeR oriented in the same direction as afeI. A similar organization of afeI-hypothetical gene-afeR has been observed in Burkholderia pseudomallei (Lewenza et al., 1999; reviewed in Gray and Garey, 2001). The function of the conserved hypothetical gene remains unknown. A candidate sigma 70-like promoter, exhibiting potential -35 and -10 consensus regions, was detected 34 bps upstream of the proposed ATG start codon of afeI. Immediately upstream of the -35 consensus region is a predicted Lux box of 20 base pairs that exhibits pseudopalindromy and conservation of nucleotide sequence with other characterized Lux boxes (Fig. 1B) (Fuqua and Winans, 1996; Whiteley and Greenberg, 2001; Schuster et al., 2004). The pseudo-palindrome can be extended for another 5 bps on either side of the predicted Lux box, making the palindrome a total of $30 \mathrm{bps}$ and superimposing it over the possible -35 promoter region (Fig. 1A).

AfeI catalyzes the formation of an unsaturated $N$-acylhomoserine lactone (AHL) of chain length $C_{14}$

In many characterized Gram-negative bacterial systems, the product of afeI is a homoserine lactone synthase that catalyzes the production of an $N$-acylhomoserine lactone (AHL) from S-adenosylmethionine (SAM) (Hanzelka and Greenberg, 1997). In order to evaluate whether the putative afeI of A. ferrooxidans also catalyzed the production of AHL, afeI was amplified by PCR and cloned into the $E$. coli expression vector pGEX-2t under the control of a tac promoter for inducible, high level expression.

Cultures of $E$. coli JM109 containing pAfeI, were extracted with dichloromethane and the extract was subjected to preparative fractionation with sep pack $\mathrm{C} 18$ as described in Methods. Five fractions were recovered
(F1-F5) and independently subjected to GC/ MS analysis. The retention times and mass spectra were compared to the retention times and mass spectra from standards of $N$ tetradecanoyl-DL-homoserine lactone (AHL$\left.\mathrm{C}_{14}\right), \mathrm{N}$-hexanoyl-DL-homoserine lactone (AHL-C 6 ) and $N$-octanoyl-DL-homoserine lactone $\left(\mathrm{AHL}-\mathrm{C}_{8}\right)$. Fraction F5 contained as main product a compound identified by retention time and mass spectrum obtained from GC/MS analysis as $\mathrm{AHL}_{14}$ (Fig. 2) suggesting that this is the major AHL product of AfeI. However, the production of minor amounts of other classes of AHL cannot be discounted. The other fractions (F1-F4) did not yield products with mass spectra of any of the standard AHLs checked (data not shown). Supernatant extracts derived from E. coli JM109 containing the cloning vector pGEX$2 \mathrm{t}$ without afel did not yield a product with mass spectrum related to AHL (data not shown) indicating that the presence of afeI is required for the production of AHL.

\section{AHL encoded by afeI has biological activity}

In order to evaluate whether the AHL derived from the expression of afeI has biological activity, E. coli JM109 pAfeI was cross-streaked on X-gal indicator agar plates with reporter strains of $S$. meliloti $\mathrm{Rm} 41$ SinI- (responds to unsubstituted AHL- $\mathrm{C}_{10-16}$ ) and A. tumefaciens NT1 (responds to AHL$\mathrm{C}_{4-14}$ ). AHL was detected by $S$. meliloti Rm41 SinI- (dark stain, streak b, Fig. 3A) but only barely by $A$. tumefaciens NT1 (streak b, Fig. 3B) indicating that the AHL produced by AfeI is biologically active in the heterologous strain S. meliloti Rm41 SinI- . Further, this activity is most likely to result from the production of an unsubstituted AHL of chain length $\mathrm{C}_{10-16}$, consistent with the results from the GC/MS experiment reported above that suggest the AHL contains a chain length of $\mathrm{C}_{14}$.

In control experiments, neither of the reporter strains respond to E. coli JM109 containing plasmid pGEX-2t (lacking afeI) showing that afeI is required (streak a, Fig. $3 \mathrm{~A}$ and $\mathrm{B}$ ). As a positive control, it can be seen that both indicator strains respond to $S$. meliloti Rm41 that produces AHLs of chain lengths $\mathrm{C}_{10-16}$ (streak c, Fig. 3A and B). 


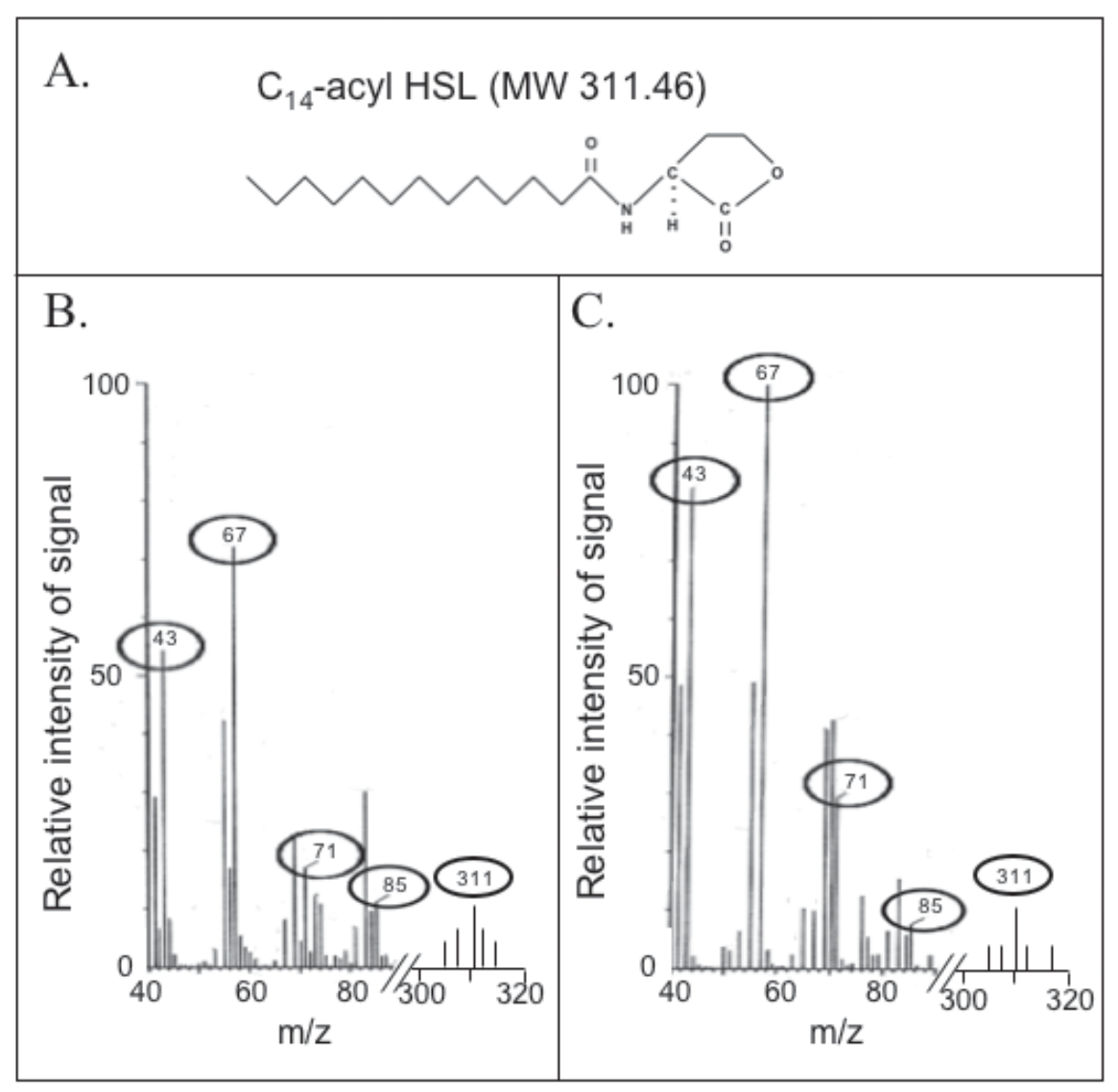

Figure 2. Identification of an acyl-homoserine lactone (AHL) whose principal chain length is $\mathrm{C}_{14}$ in the extracellular supernatant derived from a culture of E. coli JM109 expressing afeI. (A) Structure of a standard unsubstituted AHL of chain length $\mathrm{C}_{14}$. Mass spectra of (B) a supernatant extract from $E$. coli strain pAfeI and (C) a synthetic unsubstituted $\mathrm{C}_{14}$-AHL (the scale to the right of the line break in the $\mathrm{x}$ axis has been amplified 5 times to show the $\mathrm{m} / \mathrm{z}$ at 311 ).

In order to determine if the AHL produced directly by $A$. ferrooxidans is biologically active, an extract of an early stationary phase whole culture of $A$. ferrooxidans was isolated by sep-pack $\mathrm{C}_{18}$ preparative column fractionation and fraction F5 (see Methods) was applied to the reporter strain $S$. meliloti Sinl- and the induction of $\beta$-galactosidase was monitored by the Miller assay. A significant induction of $\beta$-galactosidase activity was detected (Fig. $3 \mathrm{C}, \mathrm{e})$, demonstrating that fraction F5 contains an active AHL- $\mathrm{C}_{10-16}$, consistent with the results from GC/MS. As controls, it is shown that a non-substituted AHL-C 8 standard does not induce $\beta$-galactosidase activity (Fig. 3, b), whereas both a nonsubstituted AHL-C 14 standard (Fig. 3C, c) and a crude extract of a culture of $S$. meliloti Rm41 (Fig. 3C, d) induce $\beta$-galactosidase activity. The addition of a blank without AHL resulted in an almost undetectable response (Fig. 3C, a). It is concluded that fraction F5 from an extract of a culture of $A$. ferrooxidans contains an active AHL identified by GC/MS analysis as AHL-C 14 .

Afel expression is induced by $A H L$

The question arises as to whether the predicted Lux box-promoter region of $A$. ferrooxidans can positively regulate the expression of afeI. A reporter plasmid, pAfeRI was constructed that fuses the first $120 \mathrm{bps}$ of afeI to the promoterless lacZ of plasmid pQF50. Included in the construction 
is the complete predicted afeR, together with the entire intergenic region between afeR and afeI (Fig. 4A). This region is predicted to contain the promoter and Lux box of afeI. pAfeR-I was cloned into E. coli JM109 and the resulting cells were crossstreaked on X-Gal indicator plates in close proximity to $S$. meliloti $\mathrm{Rm} 41$ producing AHLs- $\mathrm{C}_{6-16}$. The blue color that developed at the junction of the cross streaking indicates the detection of AHL by E. coli containing pAfeR-I (streak a, Fig. 4C). A control plasmid, termed pAfe $\Delta \mathrm{R}-\mathrm{I}$, was constructed that is similar to pAfeR-I but lacks the afeR gene (Fig. 4B). E. coli JM109 containing pAfe $\Delta$ R-I does not respond to exogenous AHL (streak b, Fig. 4C) indicating that the presence of afeR is required for the response. This is interpreted to mean that AHLs- $\mathrm{C}_{10-16}$ are capable of inducing transcription of the lacZ reporter gene from the afeI promoter via the activation of this promoter by LuxR, as has been demonstrated in other Gramnegative bacteria. It is also consistent with the idea that a Lux box that responds to LuxR is present in pAfeR-I.
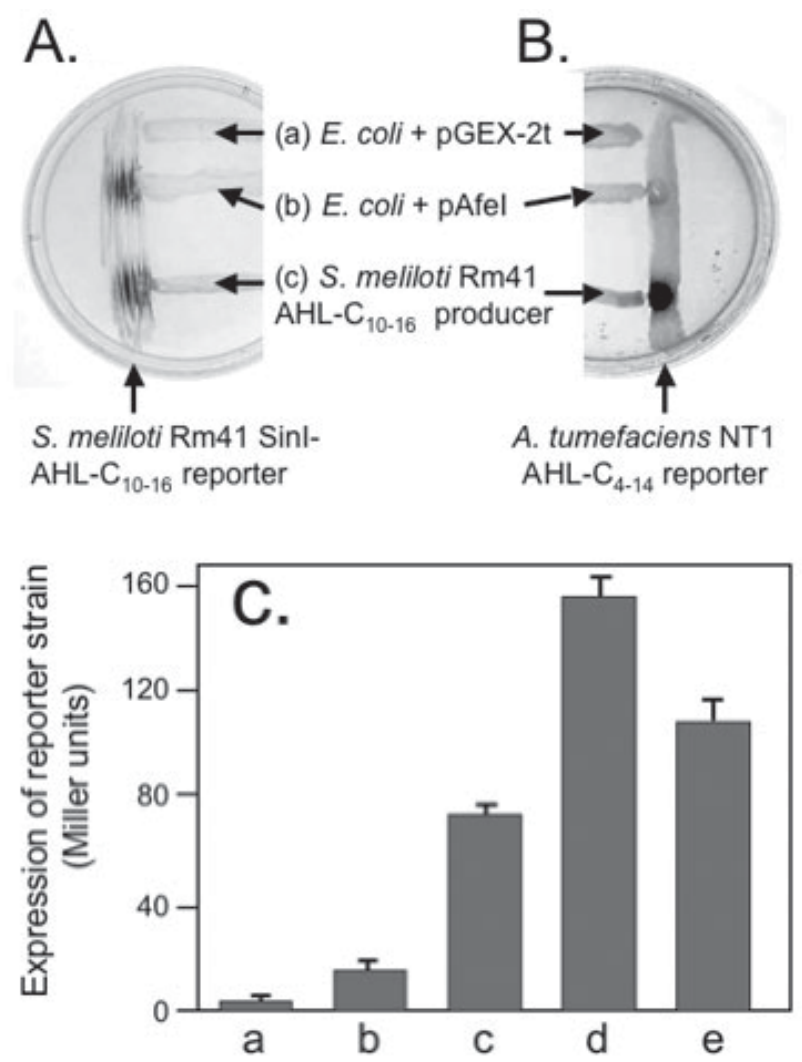

Figure 3. Biological activity of A. ferrooxidans afeI. (A) Demonstration that E. coli JM109 containing afeI is capable of inducing the expression of $\beta$-galactosidase (dark spot in streak b) in Reporter strains of (A) S. meliloti SinI- and (B) A. tumefaciens NT1 streaked with E. coli JM109 containing plasmid pGEX-2T lacking afeI (streak a), plasmid pAfeI containing afeI (streak b) or $S$. meliloti Rm41 that produces AHLs of various chain lengths from $\mathrm{C}_{10}$ to $\mathrm{C}_{16}$ Plates contain X-gal that produces a blue color (appears as a dark stains in Fig. 3) in the presence of $\beta$-galactosidase activity. (C) Measurement of $\beta$-galactosidase activity in Miller units (see Methods). S. meliloti Rm41 SinI- AHL reporter strain was grown for $6 \mathrm{~h}$ in the presence of: (a) no added AHL; (b) Unsubstituted AHL-C ${ }_{8}$; (c) Unsubstituted AHL-C 14 ; (d) crude extract from a culture of S. meliloti strain $\mathrm{Rm} 41$ producing $\mathrm{AHL}_{10-16}$, and (e) fraction $\mathrm{F} 5$ from sep-pack $\mathrm{C}_{18}$ preparative column fractionation of an extract from a culture of A. ferrooxidans. The means and standard deviations of triplicate experiments are shown. 


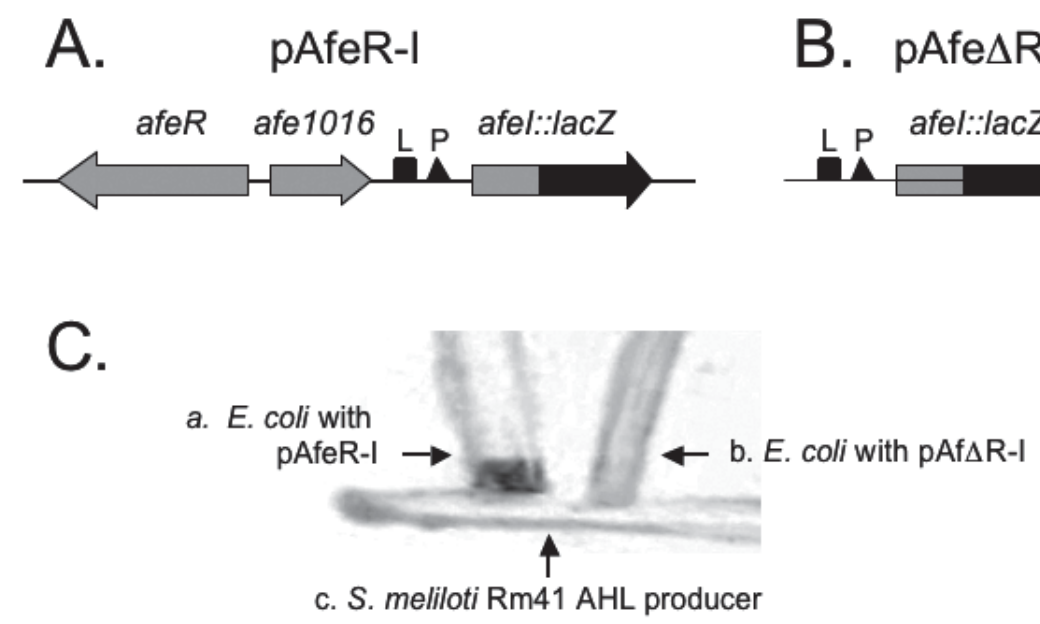

Figure 4. Demonstration that afeI expression can be activated by AHL. (A) Illustration of pAfeR-I showing the construction used for reporting the presence of exogenous AHL. L and P show the location of the predicted Lux box and putative promoter, respectively, of afeI. (B) Illustration of pAfe $\Delta$ R-I (C) E. coli JM109 containing either (a) pAfeR-I or (b) pAfe $\Delta$ R-I crossed streaked on XGal indicator plates with (c) S. meliloti Rm41 that produces AHL $\mathrm{C}_{10-16}$. The dark stain on streak (a) indicates the presence of $\beta$-galacatosidase activity.

AfeI and afe $R$ expression is maximal in early staionary phase when A. ferrooxidans is grown in sulfur

Semi-quantitative RT-PCR was used to determine the expression of afeI and afeR at three different stages, mid-log, early stationary and late stationary phases, during the culture of $A$. ferrooxidans in $9 \mathrm{~K}$ medium supplemented with sulfur. As an internal standard, $\operatorname{rec} A \mathrm{RNA}$, isolated during the three growth stages, was also amplified by semi-quantitative PCR . Expression of recA does not change during growth of $A$. ferrooxidans (Liu et al., 2000). The cDNA products resulting from RT-PCR amplification of RNA from afeI, afeR and recA at non-saturating cycles of RT-PCR were visualized by agarose gel electrophoresis and the resulting ethidium bromide stained bands were quantitated by densitrometry and image analysis. The amount of product derived from afeI and afe $R$ was plotted as a percentage relative to the amount of product derived from recA (Fig. 5A). Maximal expression of both afeI and $a f e R$ occurred in early stationary phase.

RNA was isolated from early stationary phase cultures of $A$. ferrooxidans grown in either 9K medium supplemented with sulfur or iron was amplified by RT-PCR using different numbers of cycles of amplification and the products visualized by agarose gel electrophoresis (Fig. 5B). RT-PCR amplification was simultaneously carried out on recA RNA (Fig. 5C). Whereas the amount of RT-PCR amplified product from recA does not vary when RNA is isolated from cells grown on $9 \mathrm{~K}$ supplemented with sulfur or iron, there is more product from both afeI and afeR from cells grown on $9 \mathrm{~K}$ supplemented with sulfur compared to iron.

\section{DISCUSSION}

Closely linked, but divergently oriented, genes (afeI and afeR) were found in the genome of the acidophilic proteobacterium, Acidithiobacillus ferrooxidans using bioinformatic procedures (Fig. 1). The predicted protein products of these genes are significantly similar to the LuxI and LuxR families of proteins, respectively, including conserved characteristic motifs, domains and patterns (Table III). A potential gene (afe1016) of unknown function was detected in the intergenic 
region between afeI and afeR. A potential gene (gi: 53721910) with similarity to afe1016 and located between genes of the luxI and luxR families was detected in Burkholderia pseudomallei K96243. This suggests the possibility that the function of afe 1016 might be quorum sensing related.

A candidate sigma 70 -like promoter and a possible Lux box were detected in the intergenic space between afeI and afeR (Fig. 1). The putative Lux box of $A$. ferrooxidans is predicted to be a palindrome of $20 \mathrm{bps}$ and is located immediately adjacent to, and upstream of, the candidate -35 consensus of the predicted afeI promoter. A similar juxtaposition of Lux boxes and promoters of other luxI-like genes has been observed (Schuster et al., 2004; Whiteley and Greenberg, 2001; Whiteley et al., 1999; Fuqua and Winans, 1996; Wagner et al., 2003). However, unlike these characterized
Lux boxes, the candidate A. ferrooxidans Lux box, exhibits an additional $10 \mathrm{bps}$ of palindromic sequence of which $5 \mathrm{bps}$ overlap the -35 consensus sequence. The significance of this observation remains to be determined.

When afeI is cloned and expressed in $E$. coli JM109 an AHL can be isolated from the culture supernatant identified as an unsubstituted AHL of chain length $\mathrm{C}_{14}$ by GC/MS analysis (Fig. 2), although the presence of minor amounts of other AHLs cannot be ruled out. In cross-streaking assays (Fig. 3A,B) with the AHL reporter strains $S$. meliloti SinI- and A. tumefaciens NT1, that respond respectively to unsubstituted AHL-C $\mathrm{C}_{10-16}$ or substituted AHL-C $4-14$, only the former reacts significantly to the AHL of A.ferrooxidans consistent with the idea that the major product of AfeI is an unsubstituted AHL of longer chain length.

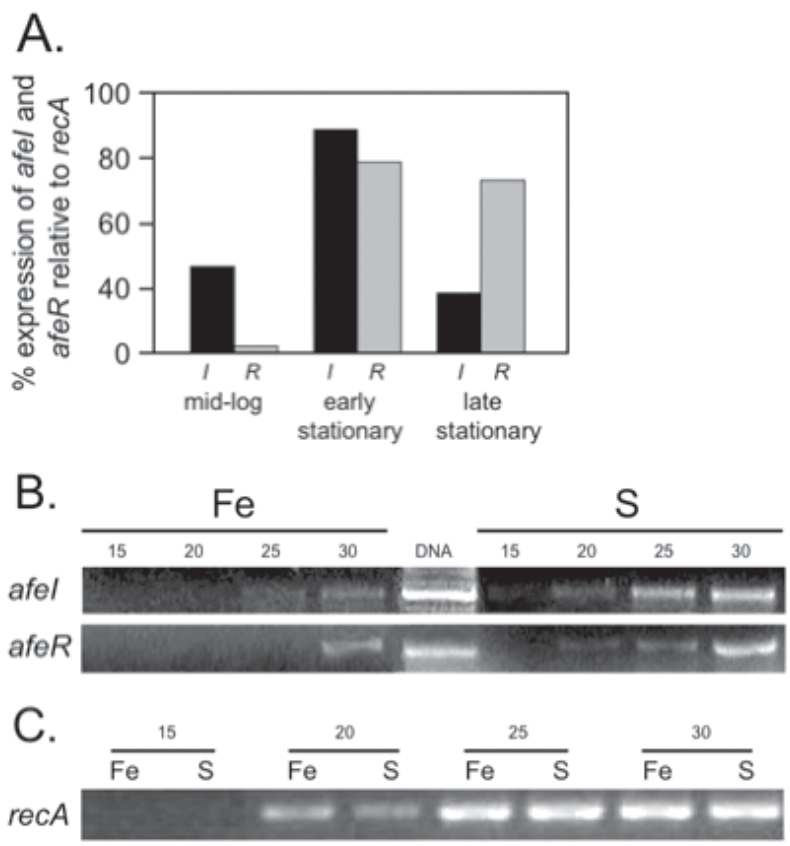

Figure 5. Determination of expression of afeI and afeR by semiquantitative RT-PCR during different phases of growth of A. ferrooxidans and from cultures grown in either $9 \mathrm{~K}$ medium supplemented with sulfur or iron. (A) $\mathrm{I}=$ afeI expression and $\mathrm{R}=a f e R$ expression. Mid-log $=$ $72 \mathrm{hrs}$, early staionary phase $=120 \mathrm{hrs}$ and late stationary phase $=240 \mathrm{hrs}$. (B) RT-PCR amplification of afeI and afeR RNA from cells grown in either 9K medium supplemented with iron (Fe) or sulfur (S). (C) RT-PCR amplification of recA RNA. (B) and (C) Numbers refer to the number of cycles of PCR amplification carried out. DNA = PCR amplification of DNA using afeI or afeR specific primers to determine the specificity of the chosen primers in the PCR reaction. 
More than ten different types of AHLs have been identified in different organisms in which an acyl chain of 4 to 18 carbon residues is attached to conserved homoserine lactone group. Additional variation occurs via substitution at the third carbon with hydroxy or oxo moieties and by the presence of double bonds in the acyl chain. Long chain AHLs have been identified in several bacteria including Paracoccus denitrificans (AHL-C $\left.{ }_{16}\right)$, Rhodobacter capsulatus (AHL- $\mathrm{C}_{14}$ and AHL-C 16 ), Rhodobacter sphaeroides (Schaefer et al., 2002), Rhizobium leguminosarum (AHL-oxo- $\mathrm{C}_{14}$ ) (Gray et al., 1996) and Sinorhizobium meliloti (AHL- $\mathrm{C}_{12}$, -oxo- $\mathrm{C}_{14}$, -oxo- $\mathrm{C}_{16: 1},-\mathrm{C}_{16: 1}$ and $\mathrm{C}_{18}$ ) (Marketon et al., 2002). The detection of long chain AHLs is complicated and their final identification usually requires radioactive assays. In the present study a combination of experiments involving $\mathrm{GC} /$ $\mathrm{MS}$ and cross-streaking in the presence of reporter strains of bacteria allowed the identification of an unsaturated AHL- $\mathrm{C}_{14}$.

According to the results of the crossstreaking assays (Fig. 3A, B), A. ferrooxidans AHL is capable of crossing the E. coli membrane. This suggests that no additional $A$. ferrooxidans gene products are absolutely required for the export of $A$. ferrooxidans AHL from E. coli. However, it remains to be determined if this is also true for its export from native $A$. ferrooxidans cells. In some organisms quorum sensing lactones cross the membrane via multidrug pumps such as MexAB and OprD (Evans et al., 1998; Kohler et al., 2001). Since active AHL can be isolated from $A$. ferrooxidans cultures, it indicates that it is stable to the low $\mathrm{pH}(\mathrm{pH}$ 3.6) of the medium. Acid stability of homoserine lactones has been previously reported (Yates et al., 2002).

The upregulation of a reporter $\beta$ galactosidase gene by exogenous AHL occured only if the reporter gene was linked to the predicted promoter and Lux box of afeI and only if luxR was present (Fig. 4). This is consistant with the well characterized model of the positive regulation of luxI by LuxR that has been described in many Gram-negative bacteria (reviewed in Pappas et al., 2004). It suggests that AfeR might mediate its regulatory role by binding to the predicted Lux box upstream of luxI as has been described in many Lux quorum sensing systems (Fuqua and Greenberg, 1998). This hypothesis has been recently experimentally validated (Barreto, M., Lefimil, C., Rivas, M., Holmes, D. and E. Jedlicki, submitted, 2005). The predicted gene af1016, of unknown function, is located in the integenic region between luxI and luxR. However, it is not known if it plays a role in quorum sensing.

Evidence from semi-quantitative real time PCR (Fig. 5A) demonstrates that both afeI and $a f e R$ are preferentially expressed in early stationary phase as has been reported for several other Gram negative bacteria (Whiteley et al., 1999). The expression of A. ferrooxidans luxI is higher than that of luxR in mid-log whereas the converse is true in late staionary phase (Fig. 5A). An interpretation of these results is that LuxR serves as a repressor of luxI in $A$. ferrooxidans. An alternative hypothesis is that the relative levels of $\operatorname{luxI}$ and $\operatorname{luxR}$ do not necessarily reflect the cellular concentrations of their respective protein products. Post-transcriptional control of LuxI levels has been demonstrated in Erwinia carotovora where it has been shown that RsmA negatively regulates synthesis of 3-oxo-C6-AHL by destabilizing the transcript encoding LuxI (Cui et al., 1995). CsrA, a member of the RsmA family of regulators, also destabilizes specific mRNAs and RsmA family members are widespread in bacteria (White et al., 1996). Regulated stability of AHL synthase transcripts may therefore be relatively common.

Both $l u x R$ and luxI exhibit increased expression in sulfur- versus iron medium (Fig. 5B). This could reflect the fact that in early stationary phase there is a higher cell population density in sulfur grown medium and this increased density might be driving the increased gene expression. Alternatively, the expression $l u x R$ and $l u x I$ might be modulated by genetic signals resulting from sensing differences in the two media. 
Given the significant amino acid similarity and conservation of protein domains, motifs and patterns of AfeI and AfeR with the LuxI and LuxR family of quorum sensing proteins and given their observed similarity of function, it is proposed that afeI and afeR are orthologs of luxI and luxR. Since the genomic organization of $l u x I$ and $l u x R$ and the predicted location of a Lux box are also conserved and since luxI expression can be induced by AHL, it is further proposed, as a testable hypothesis, that $A$. ferrooxidans contains a quorum sensing system that works according to the LuxI-LuxR paradigm. It will be important in the future to determine the genetic targets of LuxR. Initial progress in this direction has been made (Barreto, M., Lefimil, C., Rivas, M., Holmes, D. and E. Jedlicki, submitted, 2005). In addition, a number of alternate quorum sensing systems have been described in bacteria (Pappas et al., 2004) and recent evidence suggests that $A$. ferrooxidans contains a second quorum sensing system based on the HtdS paradigm (Rivas, M., Holmes, D. and E. Jedlicki, submitted, 2005). A future challenge will be to build models of the cellular behavior(s) specified by these two quorum sensing systems and to determine their activating signals and the integration of their genetic responses.

\section{ACKNOWLEDGEMENTS}

Work supported by Fondecyt No. 1010623 and 1050063. We thank the Institute of Genome Research (TIGR) and Integrated Genomics, Inc. for the use of their partial sequence of the Acidithiobacillus ferrooxidans genome. We thank Stephen Winans for providing cultures of Agrobacterium tumefaciens NT1, Juan Gonzalez for providing Sinorhizobium meliloti Rm41 and Rm41 SinI- and E. Lynn Zechiedrich for providing $E$. coli LZ2139 DsdiA::kan. We thank Myriam González from the Federico Santa María Technical University, Valparaiso, for assistance with the GC/MS experiments.

\section{REFERENCES}

BARRETO M, JEDLICKI E, HOLMES DS (2005) A gene cluster for the formation of extracellular polysaccharide (EPS) precursors in the chemolithoautotroph Acidithiobacilus ferrooxidans. Appl Environ Microbiol 71, 2907-2909.

BARRETO M, QUATRINI R, BUENO S, ARRIAGADA C, VALDÉS J, SILVER S, JEDLICKI E, HOLMES DS (2003) Aspects of the predicted physiology of Acidithiobacillus ferrooxidans deduced from an analysis of its partial genome sequence. Hydrometallurgy 71, 97-105.

BOLLINGER N, HASSETT DJ, IGELWSKI BH, COSTERTON JW, McDERMOTT TR (2001) Gene expression in Pseudomonas aeruginosa: evidence of iron override effects on quorum sensing and biofilmspecific gene regulation. J Bacteriol 183, 1990-1996.

BOND PL, DRUSCHEL GK, BANFIELD JF (2000) Comparison of acid mine drainage microbial communities in physically and geochemically distinct ecosystems. Appl Environ Microbiol 66 (11), 49624971.

CONWAY BD, VENU V, SPEERT DP (2002) Biofilm formation and acyl homoserine lactone production in the Burkholderia cepacia complex. J Bacteriol 184, 5678-5685.

CUI Y, CHATTERJEE A, LIU Y, DUMENYO CK, CHATTERJEE AK (1995) Identification of a global represor gene rsmA, of Erwinia carotovora subs. carotovora that controls extracellular enzymes, $\mathrm{N}-(3-$ oxohexanoyl)-L-homoserine lactone, and pathogenicity in soft-rotting Erwinia spp. J Bacteriol 177, 51085115 .

DEVINE JH, COUNTRYMAN C, BALDWIN TO (1988) Nucleotide sequence of the $\operatorname{luxR}$ and $\operatorname{luxI}$ genes and structure of the primary regulatory region of the lux regulon of Vibrio fisheri. Biochem 27, 837-842.

EVANS K, PASSADOR L, SRIKUMAR R, TSANG E, NEZEZON J, POOLE, K (1998) Influence of the MexAB-OprM multidrug efflux system on quorum sensing in Pseudomonas aeruginosa. J Bacteriol 180, 5443-5447.

FARAH C, BANDERAS A, JEREZ CA, GUILIANI N (2004) Searching for physiological functions regulated by the quorum sensing autoinducer AI-1 promoted by afeI/afeR genes in Acidithiobacillus ferrooxidans. pp 1361-1368 in: BioHydrometallurgy: A Sustainable Technology in Evolution. Eds: TSEZOS M, HATZIKIOSEYIAN A, REMOUDAKI E. National Technical University of Athens.

FARINHA MA, KROPINSKI AM (1990) Construction of broad-host-range plasmid vectors for easy visible selection and analysis of promoters. J Bacteriol 172, 3496-3499.

FLAVIER A, GANOVA-RAEVA L, SCHELL M, DENNY $\mathrm{T}$ (1997) Hierarchical autoinduction in Ralstonia solanacearum: control of acyl-homoserine lactone production by a novel autoregulatory system responsive to 3 -hydroxy palmitic acid methyl ester. $J$ Bacteriol 179, 7089-7097.

FRAZER KA, ELNITSKI L, CHURCH DM, DUBCHAK I, HARDISON RC (2003) Crossspecies sequence comparisons: a review of methods and available resources. Genome Res 13, 1-12.

FUQUA C, GREENBERG EP (1998) Self perception in bacteria: quorum sensing with acylated homoserine lactones. Curr Opin Microbiol 1, 183-189.

FUQUA C, WINANS SC (1996) Localization of OccRactivated and TraR-activated promoters that express 
two ABC-type permeases and the traR gene of $\mathrm{Ti}$ plasmid pTiR10. Mol Microbiol 20, 1199-1210.

GEHRKE T, TELEGDI J, THIERRY D, SAND W (1998) Importance of extracellular polymeric substances from Thiobacillus ferrooxidans for bioleaching. Appl Environ Microbiol 64, 2743-2747.

GRAY KM, GAREY JR (2001) The evolution of bacterial LuxI and LuxR quorum sensing regulators. Microbiol 147, 2379-2387.

GRAY KM, PEARSON JP, DOWNIE JA, BOBOYE BE, GREENBERG EP (1996) Cell-to-cell signaling in the symbiotic nitrogen-fixing bacterium Rhizobium leguminosarum: autoinduction of a stationary phase and rhizosphere-expressed genes. J Bacteriol 178, 372376.

GUACUCANO M, LEVICAN G, HOLMES DS, JEDLICKI E (2000) An RT-PCR artifact in the characterization of bacterial operons. Electronic J Biotech 3, 213-216.

HANZELKA BL, GREENBERG EP (1997) Quorum Sensing in Vibrio fischeri: evidence that $S$ adenosylmethionine is the amino acid substrate for autoinducer synthesis. J Bacteriol 178, 5291-5294.

HUBER B, RIEDEL K, HENTZER M, HEYDORN A, GOTSCHLICH A, GIVSKOV M, MOLIN S, EBERL L (2001) The cep quorum-sensing system of Burkholderia cepacia $\mathrm{H} 111$ controls biofilm formation and swarming motility. Microbiol 147, 2517-2528.

KELLY D, WOOD A (2000) Reclassification of some species of Thiobacillus to the newly designated genera Acidithiobacillus gen. nov., Halothiobacillus gen. nov. and Thermithiobacillus gen. nov. Int J Syst Evol Microbiol 50,511-516.

KOHLER T, VAN DELDEN C, CURTY LK, HAMZEHPOUR MM, PECHERE JC (2001) Overexpression of the MexEFOprN multidrug efflux system affects cell-to-cell signaling in Pseudomonas aeruginosa. J Bacteriol 183, 5213- 5222.

LATIFI A, FOGLINO M, TANAKA K, WILLIAMS P, LAZDUNSKI, A. (1996) A hierarchical quorumsensing cascade in Pseudomonas aeruginosa links the transcriptional activators LasR and RhIR (VsmR) to expression of the stationary-phase sigma factor RpoS. Mol Microbiol 21, 1137-1146.

LATIFI A, WINSON MK, FOGLINO M, BYCROFT BW, STEWART GSAB, LAZDUSKI A, WILLIAMS P (1995) Mutiple homologues of Lux R and Lux I control expression of virulence determinants and secondary metabolites through quorum sensing in Pseudomonas aeruginosa PAO1. Mol Microbiol 17, 333-343.

LEWENZA S, CONWAY B, GREENBERG EP, SOKOL PA (1999) Quorum sensing in Burkholderia cepacia: identification of the LuxRI homologs CepIR. J Bacteriol 181, 748-756.

LIU Z, GUILIANI N, APPYA-AYME C, BORNE F, RATOUCHNIAK J, BONNEFOY V (2000) Construction and characterization of a $\operatorname{rec} A$ mutant of Thiobacillus ferrooxidans by marker exchange mutagenesis. J Bacteriol 182 (8), 2269-2276.

LLAMAS I, KESHAVAN N, GONZÁLEZ JE (2004) Use of Sinorhizobium meliloti as an indicator for specific detection of long-chain $N$-acyl homoserine lactones. Appl Environ Microbiol 70, 3715-3723.

MARKETON MM, GRONQUIST MR, EBERHARD A, GONZÁLEZ JE (2002) Characterization of the Sinorhizobium meliloti sinR/sinI locus and the production of novel $\mathrm{N}$-acyl homoserine lactone. $J$ Bacteriol 184(20), 5686-5695.

MCCLEAN K, WINSON M, FISH L, TAYLOR A, CHHABRA S, CAMARA M, DAYKIN M, LAMB J, SWIFT S, BYCROFT B, STEWART G, WILLIAMS P
(1997) Quorum sensing and Chromobacterium violaceum: exploitation of violacein production and inhibition for the detection of $\mathrm{N}$-acylhomoserine lactones. Microbiol 143, 3703-3711.

McCUE L, THOMPSON W, CARMACK C, RYAN MP, LIU JS, DERBYSHIRE V, LAWRENCE CE (2001) Phylogenetic footprinting of transcription factor binding sites in proteobacterial genomes. Nucleic Acids Res 29, 774-782.

McGUIRE AM, HUGHES JD, CHURCH GM (2000) Conservation of DNA regulatory motifs and discovery of new motifs in microbial genomes. Genome Res 10, 744-757.

MILLER JH (1972) Experiments in molecular genetics. Cold Spring Harbor Laboratory, Cold Spring Harbor, N.Y.

MILLER M, BASSLER B (2001) Quorum sensing in Bacteria. Ann Rev Microbiol 55,163-199.

NEALSON K (1999) Early observations defining quorumdependent gene expression. pp: 277-289. In: Cell-Cell Signaling in Bacteria. DUNNY G, WINANS S (eds) ASM Press, Washington D. C.

PAPPAS K, WEINGART CL, WINANS SC (2004) Chemical communication in proteobacteria: biochemical and structural studies of signal synthases and receptors required for intercellular signaling. Mol Microbiol 53 (3), 755-769.

PEARSON JP, VAN DELDEN C, IGLEWSKI BH (1999) Active efflux and diffusion are involved in transport of Pseudomonas aeruginosa cell-to-cell signals. J Bacteriol 181, 1203-1210.

PESCI E, IGLEWSKI B (1999) Quorum sensing in Pseudomonas aeruginosa. pp:147-155. In: Cell-Cell Signaling in Bacteria. DUNNY G, WINANS S (eds) ASM Press, Washington D. C.

QUATRINI R, LEFIMIL C, HOLMES DS, JEDLICKI E (2005) The ferric iron uptake regulator (Fur) from the extreme acidophile, Acidithiobacillus ferrooxidans. Microbiol 151, 2005-2015.

RAWLINGS DE (2002) Heavy metal mining using microbes. Annu Rev Microbiol 56, 65-91.

SAMBROOK JE, FRITSCH EF, MANIATIS T (1989) Molecular cloning: a laboratory manual, $2^{\text {nd }}$ ed. Cold Spring Harbor Laboratory Press, Cold Spring Harbor, N.Y.

SCHAEFER AL, TAYLOR TA, BEATTY JT, GREENBERG EP (2002) Long-chain acyl-homoserine lactone quorum-sensing regulation of Rhodobacter capsulatus gene transfer agent production. J Bacteriol 184, 6515-6521.

SCHIPPERS A, SAND W (1999) Bacterial leaching of metal sulfides proceeds by two indirect mechanisms via thiosulfate or via polysulfides and sulfur. Appl Environ Microbiol 65, 319-321.

SCHUSTER M, URBANOWSKI ML, GREENBERG EP (2004) Promoter specificity in Pseudomonas aeruginosa quorum sensing revealed by DNA binding of purified LasR. Proc Natl Acad Sci USA 101(45), 15833-15839.

SEEGER M, CÁMARA B, HOFER B 2001. Dehalogenation, denitration, dehydroxylation and angular attack of substituted biphenyls and related compounds by a biphenyl dioxygenase. J Bacteriol 183, 3548-3555.

SEEGER M, GONZÁLEZ M, CÁMARA B, MUÑOZ L, PONCE E, MEJÍAS L, MASCAYANO C, VÁSQUEZ Y, SEPÚLVEDA-BOZA S (2003) Biotransformation of natural and synthetic isoflavonoids by two recombinant microbial enzymes. Appl Environ Microbiol 69, 50455050 
STEVENS AM, GREENBERG EP (1997) Quorum sensing in Vibrio fisheri: essential elements for activation of the luminescence genes. J Bacteriol 179, 557-562.

SWIFT S, KARLYSHEV AV, FISH L, DURANT EL, WINSON MK, CHHABRA SR, WAGNER VE, BUSHNELL D, PASSADOR L, BROOKS AI, IGLEWSKI BH (2003) Microarray analysis of Pseudomonas aeruginosa quorum-sensing regulons: effects of growth phase and environment. J Bacteriol 185, 2080-2095.

WHITE D, HART ME, ROMEO T (1996) Phylogenetic distribution of the global regulatory gene csrA among eubacteria. Gene 182, 221-223.

WHITEHEAD NA, BARNARD AM, SLATER H, SIMPSON NJ, SALMOND GP (2001) Quorum-sensing in Gram-negative bacteria. FEMS Microbiol Rev 25, 365-404.

WHITELEY M, GREENBERG EP (2001) Promoter specificity elements in Pseudomonas aeruginosa quorum-sensing-controlled genes. J Bacteriol 183, $5529-5534$.
WHITELEY MK, LEE M, GREENBERG EP (1999) Identification of genes controlled by quorum sensing in Pseudomonas aeruginosa. Proc Natl Acad sci USA 96, 13904-13909.

WINANS SC, ZHU J, MORÉ M (1999) Cell densitydependent gene expression by Agrobacterium tumefaciens during colonization of crown gall tumors. pp: 117-128. In: Cell-Cell Signaling in Bacteria. DUNNY G, WINANS SC (eds) ASM Press, Washington D. C.

YATES EA, PHILIPP B, BUCKLEY C, ATKINSON S, CHHABRA SR, SOCKETT RE, GOLDNER M, DESSAUX Y, CAMARA M, SMITH H, WILLIAMS P (2002) $\mathrm{N}$-acylhomoserine lactones undergo lactonolysis in a $\mathrm{pH}-$, temperature-, and acyl chain lengthdependent manner during growth of Yersinia pseudotuberculosis and Pseudomonas aeruginosa. Infect Immun 70, 5635-46.

YATES J, HOLMES DS (1988) Two families of repeated DNA sequences in Thiobacillus ferrooxidans. Proc Natl Acad Sci USA 85, 7284-7287. 